OPEN ACCESS

Edited by: Anna De Rosa

Università di Napoli Federico II, Italy

Reviewed by:

Gina Ferrazzano,

Sapienza University of Rome, Italy

Arushi Gahlot Saini,

Post Graduate Institute of Medical

Education and Research

(PGIMER), India

*Correspondence:

Yuehua Zhang

zhangyhdr@126.com

Specialty section:

This article was submitted to

Movement Disorders,

a section of the journal

Frontiers in Neurology

Received: 31 January 2021

Accepted: 06 April 2021

Published: 28 May 2021

Citation:

Yang $X$, Niu $X$, Yang $Y$, Cheng $M$, Zhang J, Chen J, Yang Z and Zhang $Y$ (2021) Phenotypes of GNAO1 Variants in a Chinese Cohort.

Front. Neurol. 12:662162. doi: 10.3389/fneur.2021.662162

\section{Phenotypes of GNAO1 Variants in a Chinese Cohort}

\author{
Xiaoling Yang, Xueyang Niu, Ying Yang, Miaomiao Cheng, Jing Zhang, Jiaoyang Chen, \\ Zhixian Yang and Yuehua Zhang*
}

Department of Pediatrics, Peking University First Hospital, Beijing, China

This study aimed to analyze the genotypes and phenotypes of GNAO1 variants in a Chinese cohort. Seven male and four female patients with GNAO1 variants were enrolled, including siblings of brothers. Ten different GNAO1 variants (nine missense and one splicing site) were identified, among which six were novel. All the variants were confirmed to be de novo in peripheral blood DNA. Eight $(73 \%, 8 / 11)$ patients had epilepsy; the seizure onset age ranged from $6 \mathrm{~h}$ after birth to 4 months (median age, 2.5 months). Focal seizures were observed in all eight patients, epileptic spasms occurred in six $(75 \%$, $6 / 8)$, tonic spasm in four $(50 \%, 4 / 8)$, tonic seizures in two, atypical absence in one, and generalized tonic-clonic seizures in one. Seven patients had multiple seizure types. Eight $(73 \%, 8 / 11)$ patients had movement disorders, seven of them having only dystonia, and one having dystonia with choreoathetosis. Varying degrees of developmental delay (DD) were present in all 11 patients. The phenotypes were diagnosed as early infantile epileptic encephalopathy (EIEE) in two (18\%) patients, which were further diagnosed as West syndrome. Movement disorders (MD) with developmental delay were diagnosed in two (18\%) brothers. EIEE and MD were overlapped in six (55\%) patients, among which two were diagnosed with West syndrome, one with Ohtahara syndrome, and the other three with non-specific EIEE. One (9\%) patient was diagnosed as DD alone. The onset age of GNAO1-related disorders was early infancy. The phenotypic spectrum of GNAO1 included EIEE, MD with DD, and DD alone.

Keywords: GNAO1, variant, epilepsy, movement disorder, developmental delay

\section{INTRODUCTION}

In 2013, Nakamura et al. had reported, for the first time, the identification of de novo mutations in the GNAO1 gene of four patients with early infantile epileptic encephalopathy-17 (EIEE17; 615473), and the mutations led to loss of function (1). In 2016, Ananth et al. reported GNAO1 mutations in patients of neurodevelopmental disorder with involuntary movements (NEDIM; 617493) (2). In 2017, Feng et al. demonstrated that the epileptic encephalopathy phenotype is related more to GNAO1 loss-of-function variants, while the movement disorders (MD), with or without epilepsy, were predominantly related to the gain-of-function variants (3).

The GNAO1 gene encodes the $\alpha$-subunit of the heterotrimeric guanine nucleotide-binding proteins $\left(\mathrm{G} \alpha_{0}\right)$. G proteins are composed of $\alpha, \beta$, and $\gamma$ subunits. They are highly abundant in the mammalian central nervous system and are involved in signal transduction. They mediate 
signals from a variety of important $G$ protein-coupled receptors (GPCRs) including GABAB, $\alpha 2$ adrenergic, adenosine A1 (A1R), and dopamine D2 (D2R) receptors (4). Mutations in GNAO1 and other G-protein subunits (GNAL), adenylyl cyclase (ADCY5), and cyclic nucleotide phosphodiesterase (PDE10A) had been reported previously for early-onset movement disorders (5, 6). The G-protein-cAMP pathway axis is known to play a key role in the pathophysiology of movement disorders. At cellular level, $\mathrm{G}_{0}$ orks as an inhibitor of voltage-gated $\mathrm{Ca}^{2+}$ channels and activator of inward rectifying potassium channels (7). Knockout of $\mathrm{G}_{0}$ in mice displayed complex variable neurological phenotypes including tremor, severe epilepsy, abnormal behavior, and severe motor control impairment (8).

In this study, we aimed to investigate the patients with GNAO1 variants in a Chinese cohort, and explore the genotypephenotype correlation for GNAO1.

\section{MATERIALS AND METHODS}

\section{Participants}

Patients were enrolled from the Department of Pediatrics, Peking University First Hospital, between January 2016 and August 2020. Eleven patients with pathogenic GNAO1 variants were collected and identified retrospectively. Written informed consent was obtained from the participants or their legal guardians for the publication of any potentially identifiable images or data included in this article. Information regarding seizure onset age, clinical manifestations, family history, genetic testing data, video electroencephalography (EEG), neuroimaging, and treatment were collected. Patients were followed-up at the pediatric neurology clinic or via telephone. The study was approved by the Ethics Committee of Peking University First Hospital [No: 2012 (453)]. Developmental delay of the patients was evaluated according to the developmental milestones at last follow-up.

The inclusion criteria were as follows: (a) genetic epilepsy with or without movement disorders, and (b) the proband carried pathogenic GNAO1 variants in peripheral blood DNA as per Sanger sequencing. The exclusion criteria were as follows: (a) other causes of epilepsy, such as structural, metabolic, immune, and infectious, excluding genetic factor; and (b) absence of GNAO1 variant in the peripheral blood DNA of probands.

\section{Genetic Analysis}

Mutation screening of GNAO1 (RefSeq NM_020988) was performed using epilepsy panel or whole-exome sequencing (WES). Synonymous changes and single-nucleotide polymorphisms (SNPs) with a minor allele frequency (MAF) higher than 5\% were removed (http://www.ncbi.nlm.nih.gov/ projects/SNP) from the sequencing results first. Pathogenicity of the identified variants was predicted by the MutationTaster server (http://www.mutationtaster.org/), Polymorphism Phenotyping v2 (PolyPhen-2) (http://genetics.bwh.harvard.edu/pph2/), and Sorting Intolerant from Tolerant (SIFT) (http://sift.jcvi.org/). GNAO1 variants identified in the patients were evaluated against those in the literature, ClinVar, 1000 Genomes, ExAC (http://exac.broadinstitute.org/), and gnomAD (http://gnomad. broadinstitute.org/). Variant classifications were determined according to the guidelines set out by the American College of Medical Genetics and Genomics (ACMGG). The potential pathogenic variations were validated by Sanger sequencing.

\section{RESULTS}

\section{Genetic Variants}

Eleven patients with pathogenic GNAO1 variants were collected in our cohort, of which seven were males and four females. Ten different pathogenic GNAO1 variants were identified in the 11 patients; nine were missense and one was a splicing site variant. All the variants were verified as de novo in peripheral blood DNA after parental testing. Two brothers (Patients 10 and 11) shared the same splicing site variant c.724$8 \mathrm{G}>\mathrm{A}$ (Figure 1). Six novel variants were detected, including c.136A $>$ G (p.K46E), c.687C > G (p.S229R), c.470T $>$ C (p.L157R), c.810C $>$ A (p.N270K), c.817G $>$ T (p.D273Y), and c.724-8G $>$ A (Figure 2). The variants were predicted to be deleterious using the MutationTaster server, PolyPhen-2, and SIFT tools. The new variants had not been reported in 1000 Genomes, ExAC, or gnomAD previously. Details about the genotypes of all 11 patients are listed in Table $\mathbf{1}$.

\section{Clinical Features}

In 11 patients with GNAO1 variants, eight $(73 \%, 8 / 11)$ had epilepsy, eight $(73 \%, 8 / 11)$ had movement disorders, and six $(55 \%, 6 / 11)$ had both; all patients had developmental delay. Detailed information of clinical data is listed in Table $\mathbf{1 .}$

In the eight patients with epilepsy, seizure onset age ranged from $6 \mathrm{~h}$ after birth to 4 months (median age, 2.5 months). While focal seizures were observed in all eight patients, epileptic spasms were seen in six $(75 \%, 6 / 8)$, tonic spasm in four $(50 \%, 4 / 8)$, and tonic seizures in two. Atypical absence seizures were observed in Patient 2. Generalized tonic-clonic seizures were observed in Patient 6. Seven patients had multiple seizure types. Status epilepticus was not observed in the patients.

Eight patients had clinical manifestation of movement disorders, including dystonia in all (Patients 2, 3, 5-8, 10, and 11), and choreoathetosis in one (Patient 10). Five patients (Patients 2, 3 , and 5-7) had brief focal or truncal dystonia triggered by sound stimulus or emotional disturbance. Three patients (Patients 8 , 10 , and 11) had severe and persistent involuntary movements. Patients 10 and 11 were brothers, harboring the same de novo GNAO1 variant c.724-8G>A (Figure 1). Patient 10 was born as the first child of healthy, non-consanguineous parents; at 5 months of age, motor developmental delay was observed by the parents. He had difficulties in head control and turning over. Independent walking was achieved at the age of 2 years, and he would walk sometimes unstably with tumble. He subsequently developed involuntary dystonia and choreoathetosis of the limbs. He had abnormal posture while walking. Symptoms disappeared when he was asleep. He had dysarthrosis and could not speak even at 16 years of age, as per last follow-up. His intellectual delay was mild. His pediatric neurologist had diagnosed him with movement disorders combined with developmental delay. His younger brother (Patient 11) also exhibited motor developmental 


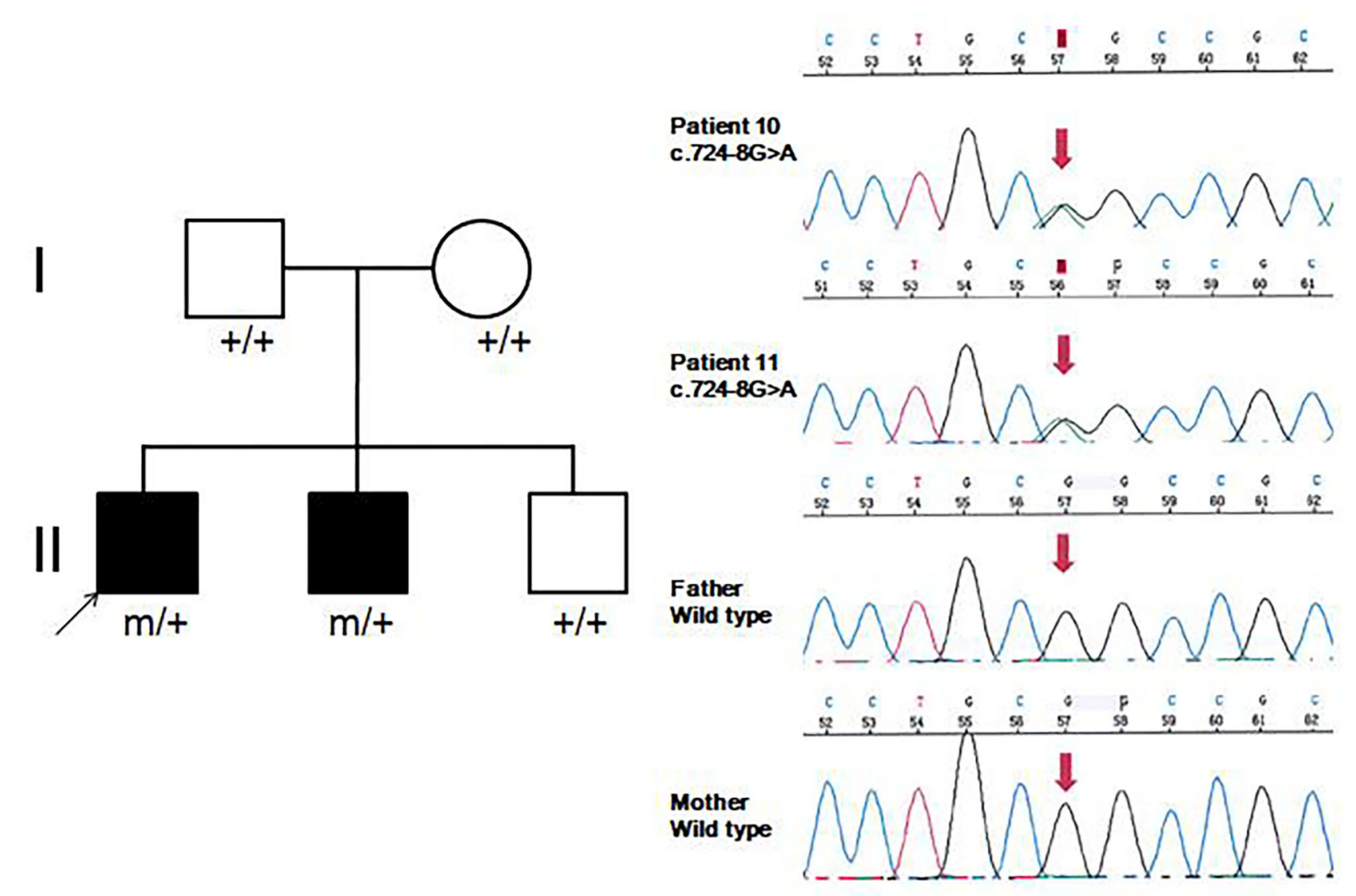

FIGURE 1 | Family pedigrees and sequence chromatograms of the two brothers. The proband and his younger brother (Patients 10 and 11) were both identified with the c.724-8G>A variant, which was further verified as de novo in peripheral blood DNA.

delay at 4 months of age. He had dystonia at 3 years of age, although no choreoathetosis was seen at last follow-up at the age of 7 years.

All patients had developmental delay and hypotonia. Among the eight patients with epilepsy, the degree of DD was significantly severe in both motor and intellectual disabilities. They could not raise their head stably, or roll over, rarely grasped objects, and had little or no response to sound or light stimuli. In the two brothers (Patients 10 and 11) with MD, motor and intellectual delay was mild. Patient 9 had no epilepsy and movement disorder. He only manifested as DD, and had severe delayed milestones and intellectual disabilities.

All the 11 patients underwent video EEG monitoring at least once. Out of the eight patients with epilepsy, EEG results showed three (Patients 1, 4, and 6) to have slow rhythm of background activity. All the eight patients with epilepsy had epileptiform discharges during the interictal phase (Figure 3). Hypsarrhythmia was observed in five patients (Patients 1-5). Two (Patients 3 and 5) revealed a suppression-burst pattern. Focal or multifocal epileptiform discharges were detected in all eight patients with epilepsy. Clinical seizures were recorded in six out of eight patients. Epileptic spasms and tonic spasms were recorded in four patients. Focal seizures were monitored in two patients. Tonic, generalized tonic-clonic, and atypical absence seizures were monitored in each patient. In Patient 9 with developmental delay only, EEG was normal. In the two brothers (Patients 10 and 11), EEG showed slow background activity, and no epileptic discharge.

All patients had undergone brain MRI. Their age at the time of MRI ranged from 1 month to 7 years. In Patient 3, brain MRI displayed white matter myelination delay at 1 year and 5 months of age. Slightly wider bilateral frontotemporal extracerebral space occurred in three patients (Patients 4, 7, and 9). White matter developmental delay was also observed in Patient 9. Brain MRI results were normal in a total of seven patients $(64 \%, 7 / 11)$.

The phenotypes were diagnosed as EIEE in two $(18 \%, 2 / 11)$ patients (Patients 1 and 4) and were further classified as West syndrome. The two brothers $(18 \%, 2 / 11)$ were diagnosed with MD with DD. EIEE and MD were overlapped in six $(55 \%, 6 / 11)$ patients, among which two were diagnosed with West syndrome (Patients 2 and 3), one with Ohtahara syndrome (Patient 5), and another three with non-specific EIEE (Patients 6, 7, and 8). One $(9 \%, 1 / 11)$ patient was diagnosed with developmental delay only (Patient 9).

In the last follow-up of, eight patients had epilepsy, seven had tried two or more antiepileptic medications, and one (Patient 8) 


\section{EIEE}

\section{MD}

\section{EIEE and MD} DD

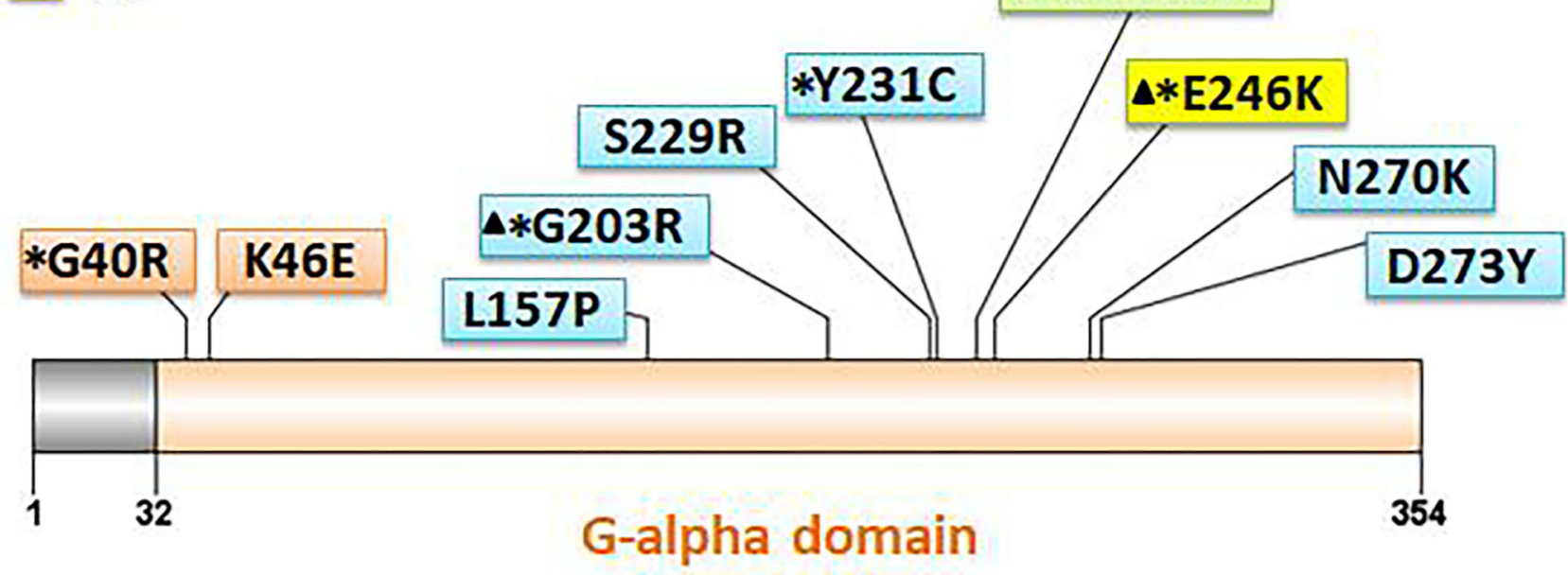

FIGURE 2 | Location of 10 GNAO1 variants in the Gao amino acid sequence. All variants are indicated corresponding to their locations within the gene. Variants with asterisks had been reported previously, and those with triangles are hotspot variants. Different background color of the box represents different phenotypes (orange: EIEE, early infantile epileptic encephalopathy; green: MD, movement disorder; blue: EIEE and MD; yellow: DD, development delay).

used only monotherapy. Seizures were well-controlled for 3-7 months in five patients. Five patients (Patients 3, 4, and 6-8) out of eight patients were seizure-free. Seizures were well-controlled with single topiramate in Patients 4 and 8 , single vigabatrin in Patient 3, add-on therapy of levetiracetam in Patient 6, and addon therapy of vigabatrin in Patient 7 . Of the eight patients with MD symptoms, three (Patients 8, 10, and 11) were treated with levodopa, trihexyphenidyl, or clonazepam, but did not respond very well, and involuntary movements persisted. However, the hypertonia of limbs was mitigated to some extent. The last followup age of our cohort ranged from 8 months to 16 years. Three patients $(27 \%)$ died before the last follow-up. Patient 1 died at 1 year and 11 months during sleep, presumably due to sudden unexpected death in epilepsy. Patient 8 died of milk choking and asphyxia at 10 months. Patient 9 died due to severe pneumonia and respiratory failure at the age of 2 years and 3 months.

\section{DISCUSSION}

Since 2013, GNAO1-associated neurological disorders have been consistently reported. GNAO1 variants could cause multiple neurodevelopmental phenotypes, including epileptic encephalopathy, involuntary movements, and developmental delay. Kelly et al. had reported 14 patients with GNAO1 variants, among which nine (64\%) had epilepsy, nine (64\%) had movement disorders, and five (36\%) had both symptoms (9). Movement disorders included chorea, dystonia, dyskinesia, stereotypes, and ataxia. One patient with p.R209C only manifested developmental delay and hypotonia, but no seizure and movement disability. In our cohort of 11 patients, eight (73\%) had epilepsy, eight (73\%) had movement abnormalities, and six (55\%) had both. One patient displayed developmental delay alone. All our patients had varying degrees of DD manifestations. GNAO1-related neurological phenotypes were of broad spectrum, including epilepsy, movement disorders, developmental delay, and combinations of all phenotypes.

Approximately 25 different GNAO1 variants had been reported previously from more than 50 patients, including 23 (92\%) missense, one frameshift deletion, and one splicing site variant (10). There are three mutation hotspots (G203, R209, and E246) in the GNAO1 gene. A genotype-phenotype correlation was also noted recently (8). Many functional studies had been performed, especially for recurrent variants. Gain-offunction variants turned out to be associated with movement disorder, while loss-of-function variants were more related to epilepsy phenotype (3). However, the relationship of variant functionality with phenotype could not explain all the cases. The explicit genotype-phenotype correlations and mechanistic basis for heterogeneity still remain to be clarified. Further studies about phenotypes, genotypes, and molecular pathways would be required for evaluating the possible genotype-phenotype correlations better. In our current study, 10 different pathogenic GNAO1 variants were identified, of which nine (90\%) were missense, and one was a splicing site variant. All were verified as de novo in peripheral blood DNA. In previous reports, siblings sharing the same GNAO1 variant and parental mosaicism 


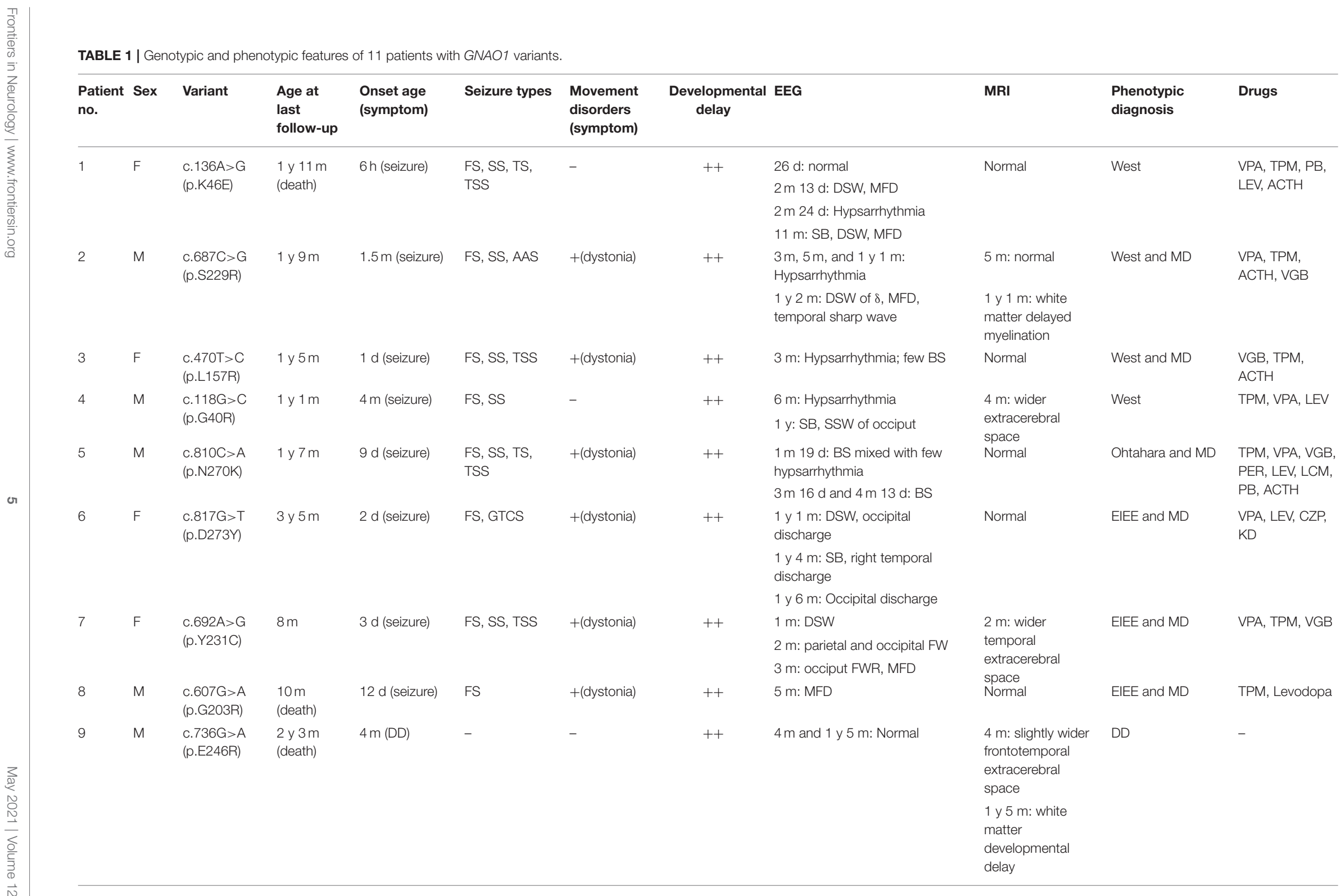




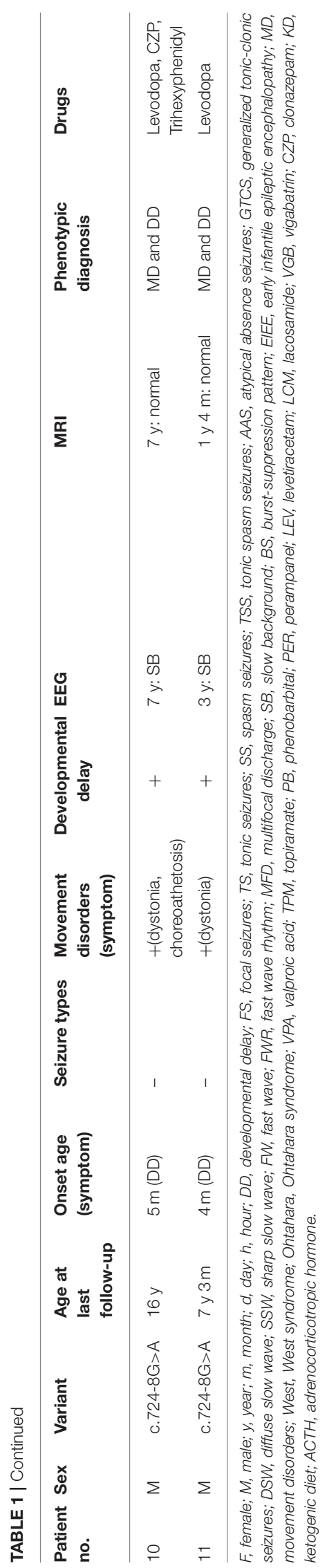

of this gene had been confirmed in a few families $(9,11$, 12). WES sequencing, performed for both siblings and their parents revealed a pathogenic variant in GNAO1 at c.119G > A (p.G40E) that appeared to be de novo in each sibling. However, re-evaluation of parental WES data revealed three variant alleles out of 150 reads (2\%) in one parent, consistent with germline mosaicism (9). Amplicon sequencing verified maternal mosaicism in one family. In this family, the patient carried the variant p.R209H, and Sanger sequencing analysis revealed a low heterozygous peak of the mother. This verification was crucial for genetic counseling in this family (12). In our study, the same variant (c.724-8G $>$ A) was identified in siblings (Patients 10 and 11 ). We highly suspected parental germline mosaicism with lowlevel frequency. Further amplicon-deep sequencing detection for the mosaic variant frequency in multiple tissue samples needs to be performed in the future. This would be very important for genetic counseling.

Epilepsy is the major phenotype of the GNAO1 gene variant. Previous studies had reported the onset of seizures to either precede or follow the MD manifestation (13). In the 22 patients reported with epilepsy (9), median age of seizure onset was 5 months (range, 1 month to 10 years); nine patients presented with EIEE, including two cases of Ohtahara syndrome and two of West syndrome. Seven patients exhibited focal seizures, while three had generalized tonic-clonic seizures. In the remaining three patients, the epileptic syndrome was not specified. In our eight patients with epilepsy, the age of seizure onset ranged from $6 \mathrm{~h}$ after birth to 4 months. Multiple seizure types were observed in most patients. The common seizure types were focal and epileptic spasms in our patients. The epileptic syndromes included West syndrome, Ohtahara syndrome, and non-specific EIEE. Feng et al. had reported that seizures were well-controlled by medication in half of the patients with epilepsy and GNAO1 variants (10). Responses to topiramate and levetiracetam had been reported earlier (10). Among our eight patients with epilepsy, seizures were completely controlled in five by topiramate, vigabatrin, and levetiracetam treatment. Results from published literature and our current study suggested that treatment with topiramate, levetiracetam, or vigabatrin should be recommended for patients with epilepsy and GNAO1 variants.

Movement abnormalities were also the major presenting symptoms of patients with GNAO1 variants. The median age at $\mathrm{MD}$ onset was 4 years, ranging from 3 months to 8 years, as per published reports (13). Most patients had a mixed hyperkinetic syndrome, encompassing different combinations of dystonia, choreoathetosis, and dyskinetic movements (13). In the overall cohort, dystonic features were most frequently reported to be a component of the MD $(65 \%, 30 / 46)$, followed by dyskinesias $(63 \%, 29 / 46)$ and choreoathetosis $(58 \%, 27 / 46)$. Eight patients in our cohort had MD; its range included dystonia and choreoathetosis. The most common feature of MD in our patients was dystonia. The last follow-up age of our patients was $<4$ years, and we believe that they should be followedup further for their clinical manifestations, including movement disorders. EEG showed low background activity but no abnormal discharge in patients with only MD (Patients 10 and 11). Partial patients obtained satisfactory management of the MD 

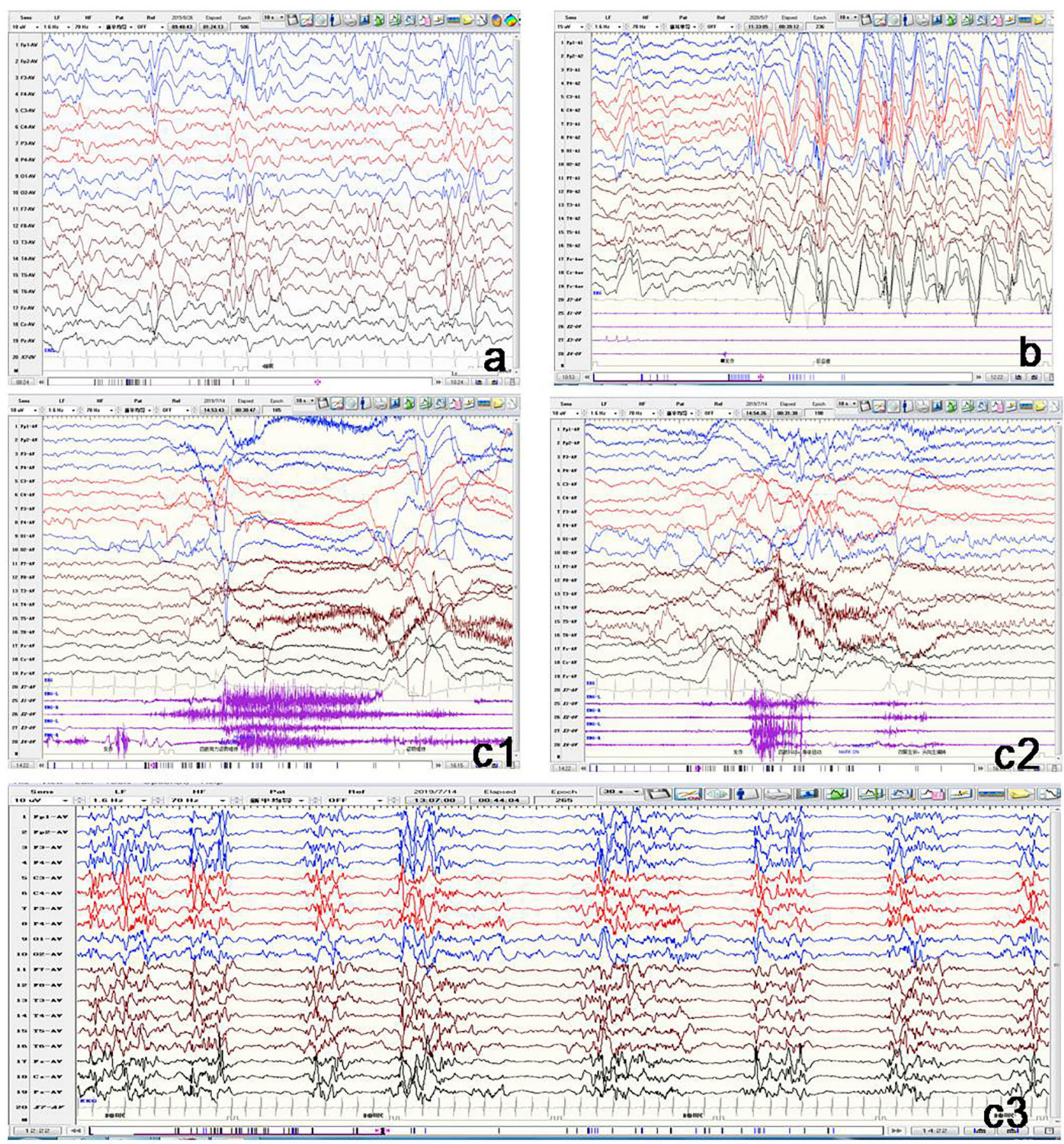

FIGURE 3 | Representative electroencephalogram (EEG) of three patients with GNAO1 variants. (a) Patient 1: Interictal EEG showing hypsarrhythmia. (b) Patient 2 : Ictal EEG of atypical absence seizures monitored by generalized high-amplitude $1.5 \mathrm{~Hz}$ spike slow wave for several seconds. (c) Patient 5: c1: Ictal EEG of tonic spasm seizures showing widespread high-amplitude sharp wave, slow wave, and fast wave with a broad voltage reduction lasting several seconds. c2: Ictal EEG of focal seizures initiated from left occipital and posterior temporal regions. c3: Interictal EEG demonstrating suppression-burst pattern.

with oral drugs, the effects being variable across the different cases. They were treated with phenobarbital, benzodiazepines (clonazepam and diazepam), levodopa, neuroleptics (haloperidol and risperidone), and antiepileptics (topiramate, carbamazepine, valproate) $(2,11,14-16)$. In one case of severe $\mathrm{MD}$ with the GNAO1 variant, the episodes were treated successfully with gabapentin (17). However, few patients presenting with severely medical-refractory MD required surgical treatment. Deep brain 
stimulation (DBS) was performed to reduce the frequency and severity of MD exacerbations (13). Three of our patients were administered oral drugs of levodopa, trihexyphenidyl, or clonazepam for controlling the MD symptoms; however, the effects were not satisfactory. DBS may be suggested and tried in such patients with refractory persistent dystonia.

Together with epilepsy or involuntary movements, the phenotypes involved global retardation of motor and intellectual development in patients with GNAO1 variants. Kelly et al. reported all 14 patients to have developmental delay and show a broad spectrum of severity (9). Five (36\%) patients showed profound impairment and were non-verbal and non-ambulatory. Few patients had the mildest developmental abnormalities, including delayed speech and motor development and mild intellectual disability. All our 11 patients had developmental delay ranging from mild to severe. In patients with epilepsy, the developmental disability was global retardation involving motor and intellectual delay. The progress was still slow in patients with controlled seizure. In two patients with MD and seizure unaffected, their motor developmental difficulties were significantly severe, and intellectual delay was milder. In our cohort, Patient 9 with GNAO1 variant, p.E246K had the phenotype of DD alone. Age at the last follow-up was 2 years and 3 months. No symptom of seizure and movement disorder was observed in this patient, although the global developmental delay was significantly severe. Regrettably, he died due to respiratory failure and sepsis after pulmonary infection. Twelve patients with the variant p.E246K had been reported previously $(2,11,18)$, and the variant was located in a mutational hot spot. For available detailed information in eight patients, seven patients had MD, and only one patient had focal epilepsy. The data led to the speculation that the p.E246K variant may be a specific site for MD phenotypes, usually without epilepsy.

The phenotypic spectrum of GNAO1-related neurodevelopmental disorders ranges broadly from epileptic encephalopathy, movement disorders, and developmental delay, to the combination of various phenotypes. De novo missense variants were frequent in the GNAO1 gene. The age of seizure onset, in the presence of the GNAO1 variant was early infancy. Dystonia may be the most common symptom related to movement disorders. When patients with EIEE had combined dystonia, GNAO1 may be suspected as the causative gene. All

\section{REFERENCES}

1. Nakamura K, Kodera H, Akita T, Shiina M, Kato M, Hoshino H, et al. De novo mutations in GNAO1, encoding a Gao subunit of heterotrimeric G proteins, cause epileptic encephalopathy. Am J Hum Genet. (2013) 93:496505. doi: 10.1016/j.ajhg.2013.07.014

2. Ananth AL, Robichaux-Viehoever A, Kim YM, Hanson-Kahn A, Cox R, Enns GM, et al. Clinical course of six children with GNAO1 mutations causing a severe and distinctive movement disorder. Pediatr Neurol. (2016) 59:81-4. doi: 10.1016/j.pediatrneurol.2016.02.018

3. Feng H, Sjögren B, Karaj B, Shaw V, Gezer A, Neubig RR. Movement disorder in GNAO1 encephalopathy associated with gain-of-function mutations. Neurology. (2017) 89:762-70. doi: 10.1212/WNL.0000000000 004262 patients had developmental delay with a wide range of severity; however, the genotype-phenotype correlations are still unclear. Further studies on genotype, phenotype, and molecular pathways would be required for better understanding of the process.

\section{DATA AVAILABILITY STATEMENT}

The datasets presented in this study can be found in online repositories. The names of the repository and accession numbers can be found below: ClinVar database and SCV001486211SCV001486219, SCV001499870.

\section{ETHICS STATEMENT}

The studies involving human participants were reviewed and approved by the Ethics Committee of Peking University First Hospital [No: 2012 (453)]. Written informed consent to participate in this study was provided by the participants' legal guardian/next of kin. Written informed consent was obtained from the individual(s), and minor(s)' legal guardian/next of kin, for the publication of any potentially identifiable images or data included in this article.

\section{AUTHOR CONTRIBUTIONS}

$\mathrm{XY}$ and YZ jointly conceived the study and contributed to the study design, initial writing, and revision of the manuscript. XN, YY, MC, JZ, JC, ZY, and YZ collected the patient data. All authors contributed to the article and approved the submitted version.

\section{FUNDING}

This work was supported by the Key Research Project of the Ministry of Science and Technology of China (Grant Nos. 2016YFC0904400 and 2016YFC0904401).

\section{ACKNOWLEDGMENTS}

We thank the patients and their family members for participating in this study. We also thank those who assisted in the data collection and Dr. Xiaodong Wang, Cipher Gene Ltd., for her assistance in manuscript editing.

4. Garcia-Marcos M, Ghosh P, Farquhar MG. Farquhar: molecular basis of a novel oncogenic mutation in GNAO1. Oncogene. (2011) 30:26916. doi: 10.1038/onc.2010.645

5. Mencacci NE, Erro R, Wiethoff S, Hersheson J, Ryten M, Balint B, et al. ADCY5 mutations are another cause of benign hereditary chorea. Neurology. (2015) 85:80-8. doi: 10.1212/WNL.0000000000001720

6. Diggle CP, Sukoff RS, Popiolek M, Hinttala R, Schülke JP, Kurian MA, et al. Biallelic mutations in PDE10A lead to loss of striatal PDE10A and a hyperkinetic movement disorder with onset in infancy. Am J Hum Genet. (2016) 98:735-43. doi: 10.1016/j.ajhg.2016.03.015

7. Colecraft HM, Brody DL, Yue DT. G-protein inhibition of $\mathrm{N}$ - and P/Q-type calcium channels: distinctive elementary mechanisms and their functional impact. J Neurosci. (2001) 21:1137-47. doi: 10.1523/JNEUROSCI.21-04-01137.2001 
8. Jiang M, Gold MS, Boulay G, Spicher K, Peyton M, Brabet P, et al. Multiple neurological abnormalities in mice deficient in the G protein Go. Proc Natl Acad Sci USA. (1998) 95:3269-74. doi: 10.1073/pnas.95.6.3269

9. Kelly M, Park M, Mihalek I, Rochtus A, Gramm M, Pérez-Palma E, et al. Spectrum of neurodevelopmental disease associated with the GNAO1 guanosine triphosphate-binding region. Epilepsia. (2019) 60:40618. doi: 10.1111/epi.14653

10. Feng H, Khalil S, Neubig RR, Sidiropoulos C. A mechanistic review on GNAO1-associated movement disorder. Neurobiol Dis. (2018) 116:13141. doi: 10.1016/j.nbd.2018.05.005

11. Schorling DC, Dietel T, Evers C, Hinderhofer K, Korinthenberg R, Ezzo D, et al. Expanding phenotype of de novo mutations in GNAO1: four new cases and review of literature. Neuropediatrics. (2017) 48:3717. doi: 10.1055/s-0037-1603977

12. Kim SY, Shim Y, Ko YJ, Park S, Jang SS, Lim BC, et al. Spectrum of movement disorders in GNAO1 encephalopathy: in-depth phenotyping and case-bycase analysis. Orphanet J Rare Dis. (2020)15:343. doi: 10.1186/s13023-02001594-3

13. Schirinzi T, Garone G, Travaglini L, Vasco G, Galosi S, Rios L, et al. Phenomenology and clinical course of movement disorder in GNAO1 variants: results from an analytical review. Parkinsonism Relat Disord. (2019) 61:19-25. doi: 10.1016/j.parkreldis.2018.11.019

14. Arya R, Spaeth C, Gilbert DL, Leach JL, Holland KD. GNAO1-associated epileptic encephalopathy and movement disorders: c.607G $>A$ variant represents a probable mutation hotspot with a distinct phenotype. Epileptic Disord. (2017) 19:67-75. doi: 10.1684/epd.2017.0888
15. Sakamoto S, Monden Y, Fukai R, Miyake N, Saito H, Miyauchi A, et al. A case of severe movement disorder with GNAO1 mutation responsive to topiramate. Brain Dev. (2017) 39:439-43. doi: 10.1016/j.braindev.2016.11.009

16. Marcé-Grau A, Dalton J, López-Pisón J, García-Jiménez MC, Monge-Galindo L, Cuenca-León E, et al. GNAO1 encephalopathy: further delineation of a severe neurodevelopmental syndrome affecting females. Orphanet J Rare Dis. (2016) 11:38. doi: 10.1186/s13023-016-0416-0

17. Akasaka M, Kamei A, Tanifuji S, Asami M, Ito J, Mizuma K, et al. GNAO1 mutation-related severe involuntary movements treated with gabapentin. Brain Dev. (2021) 43:576-79. doi: 10.1016/j.braindev.2020.12.002

18. Saitsu H, Fukai R, Ben-Zeev B, Sakai Y, Mimaki M, Okamoto N, et al. Phenotypic spectrum of GNAO1 variants: epileptic encephalopathy to involuntary movements with severe developmental delay. Eur J Hum Genet. (2016) 24:129-34. doi: 10.1038/ejhg.2015.92

Conflict of Interest: The authors declare that the research was conducted in the absence of any commercial or financial relationships that could be construed as a potential conflict of interest.

Copyright (C) 2021 Yang, Niu, Yang, Cheng, Zhang, Chen, Yang and Zhang. This is an open-access article distributed under the terms of the Creative Commons Attribution License (CC BY). The use, distribution or reproduction in other forums is permitted, provided the original author(s) and the copyright owner(s) are credited and that the original publication in this journal is cited, in accordance with accepted academic practice. No use, distribution or reproduction is permitted which does not comply with these terms. 